\title{
Morphophonology of Mengrelian Preverbal Markers and Structure of PF
}

\author{
Leila Lomashvili (Corresponding author) \\ Department of English and Humanities, Shawnee State University \\ 940 Second Street, Portsmouth, OH, USA \\ E-mail: 1lomashvili@shawnee.edu
}

Doi:10.7575/aiac.alls.v.6n.1p.172

Received: 28/09/2014

URL: http://dx.doi.org/10.7575/aiac.alls.v.6n.1p.172

Accepted: 14/12/2014

\begin{abstract}
The paper examines the preverbal markers in Mengrelian as the possible loci of post-syntactic phonological rules that affect the allomorphy of these preverbs. The goal of the paper is to pinpoint the series of these rules and the extent to which the final form of the derived material changes as a result of their application. The phonological rules are examined in light of cyclic and post-cyclic processes that have different local domains of application such as nested constituents for the former and entire words for the latter (Halle \& Nevins, 2009). It is shown that that phonological rules may obscure the derivation of surface forms from underlying representations and that the meaning of preverbal markers may not be kept as a result of these changes. The paper concludes that the derivational view of morphology such as Distributed Morphology (Halle \& Marantz, 1993) should be modified to accommodate these data.
\end{abstract}

Keywords: cyclic, post-cyclic, phonological rule, preverb, PF

\section{Introduction}

The paper utilizes the Distributed Morphology framework (DM) in the account of the phonological processes affecting Mengrelian preverbal markers (Halle \& Marantz 1993, 1994). The main goal of research is to come up with the theory that accounts for the interaction of various phonological rules affecting the PF branch in the derivation of verbal expressions. The paper draws on the empirical data presented in Kajaia (2001) and Danelia (1942) as well as native speaker consultations for the purposes of this research on preverbal markers.

\subsection{Structure of grammar in DM}

In DM, the post-syntactic component of grammar is where the main source of variation among the possible allomorphs of morphemes is located. This can be due to various phonological processes affecting the derivation of words at various points of Phonological Form (PF). The PF in this account like in many others' (Arregi and \& Nevins, 2012 among others) includes not only morphological processes such as fusion, fission and impoverishment affecting morphosyntactic features and nodes but also the phonological rules that affect the phonological realization of constituents. The following architecture of grammar in DM is of relevance for the location of these rules:

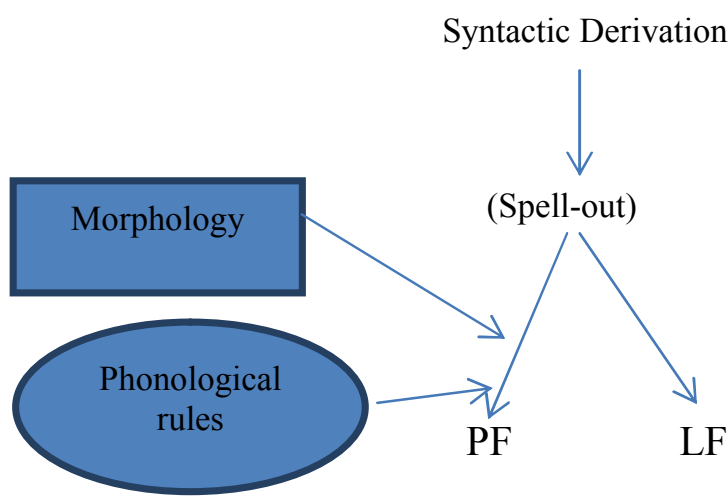

Figure 1. Architecture of grammar

The figure represents the structure of Spellout as consisting of the two types of processes: first, morphological operations affecting the morpho-syntactic feature specifications such as impoverishment, fission, fusion, etc. and the second, phonological processes per se. The exact locus of the phonological processes is not of concern in this paper, but it is argued that these processes affect verbal constructions presumably after the Linearization and Vocabulary Insertion (VI) apply to these constituents. (note 1) 
The timing of application of various phonological rules is also of importance in accounting for the surface forms (SFs) of complex morphological units. Whether the rules apply in cyclic or post-cyclic blocks may be relevant for the morphotactics of the derived word (Halle \& Mohanan, 1985; See also Halle \& Matushansky, 2006; Halle \& Nevins, 2009). The rules of cyclic block apply to each nested constituent at a time while post-cyclic rules apply once to an entire word without regard to the morphological constituency (Halle \& Matushansky, 2006). In addition, I propose in this paper that cyclic rules affect the meaning of individual constituents and a word as a whole while post-cyclic rules do not. This type of interaction between cyclic/post-cyclic rules and the meaning of morphological constituents indicates that cyclic phonological rules apply after Linearization and VI takes place, i.e. when the morpheme order and their phonological realization is determined at PF.

\subsubsection{Locus of phonological rules and their interactions}

The following notational convention is also adopted from the DM literature for the Phonological Exponent (PE) of the preverbal markers as the realization of the terminal node with the Morphosyntactic Feature Specification (MFS) matched by certain contexts since these PEs usually carry aspectual information:

(1) $\mathrm{x} \leftrightarrow[\mathrm{T} / \mathrm{Asp},-$ Present $] /$ $\mathrm{V}$

This convention shows that the preverbal markers of viewpoint aspect apply in the contexts where the Tense can only have past or future reference to denote the completed event with the endpoint (Verkyul, 1989). The same markers may also indicate the directionality of events denoted by the Root, and other additional morphemes such as assertive and negative markers may also be added in the preverbal component of the template. The relevant details will be discussed below, in Section 1.2.

Another important premise for the analysis of the morphotactics of preverbal markers is that the Linearization of morphemes precedes the VI since the linear order constrains the context in which particular exponents are inserted, and even the morphological context for the insertion of exponents is constrained by the linear adjacency of morphemes. In many cases, linear adjacency may coincide with the structural adjacency (sisterhood of these nodes); however, this may not always be a case. Some of the nodes realized by the linearly adjacent morphemes may not be sisters in syntax as shown in the following example of aspectual morphemes and the assertive marker:

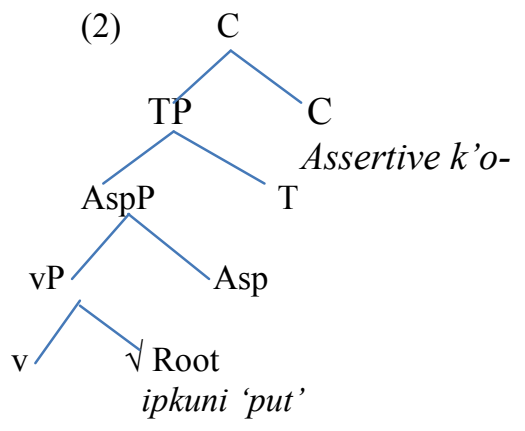

As seen in this tree, the $\mathrm{Asp}^{0}$ head is not in the sisterhood relation with the Complementizer head realized as the assertive particle $k^{\prime} o$ - at the beginning of various verbs but in an actual verbal form such as (3) the assertive and aspectual markers are linearly adjacent. This paper does not go into the details of all post-syntactic processes that are responsible for the linear order of morphemes attested in the following complex forms since it is not a goal here to give a comprehensive account of morphotactics. The main idea to keep in mind is that linear adjacency may not always parallel the structural one:

(3) k’o-mi-k'[o]-ipkuni

\section{Assert-Asp-Assert-put}

'he even put it on' (Kajaia, 2001, p.62)

\subsubsection{Interaction between phonological rules}

The paper utilizes the derivational model of phonology for expository purposes to account for the SFs of preverbal markers and also due to the fact that it is justified by the derivational framework of morphology adopted here (Chomsky \& Halle, 1968; Kenstowicz \& Kisseberth, 1979). First, this framework is in accord with our general claim that the modules of grammar such as syntax and (post-syntactic) morphology are derivational. Second, a derivational theory of phonology best explains the opaque interactions between various phonological rules resulting in the SFs that may not be recognizable from their underlying representations (URs). The interaction between these rules, including selfdestructive feeding is illustrated in Section 2.3 of this paper (Bacovič, 2007).

Another classification criteria introduced with respect to the phonological rules is whether a) they apply to certain morphemes in specific morphological contexts and are referred to as morpheme-specific phonological rules (Arregi \& Nevins, 2012) or b) they are related to the syllabification constraints imposed by language-specific syllabification requirements. Both morpheme-specific and syllable structure-affecting rules may apply to the same word and they will be separately analyzed in Sections $2.1,2.2$, and 2.3 . 
I also utilize feeding/bleeding relations between various phonological rules in the derivation of SFs. Gussenhoven \& Jacobs' (2011) definition of natural rule ordering developed from Kiparsky's (1968) rule interactions is referred to for the analysis of phonological rule interactions. For example, when the rule A increases the forms to which rule B applies, the order A-B is feeding order while the order of rules B-A in which A increases the forms to which rule the rule B applies will be referred to as a counter-feeding order (Gussenhoven \& Jacobs, 2011, p. 114). The example of feeding rule is the English preglottalization which glottalizes voiceless plosives in coda position fed by the fortis stop insertion which inserts voiceless stops between the nasal and the fricative consonant:

Table 1. Example of feeding rule

\begin{tabular}{|c|c|c|c|}
\hline Underlying & Fortis Stop & Preglottalization & Written Forms \\
\hline prins & prints & prin?ts & prince \\
\hline $\operatorname{l\varepsilon \eta } \theta$ & $\operatorname{l\varepsilon \eta \mathrm {k}\theta }$ & 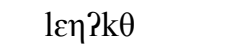 & length \\
\hline wo:m $\mathrm{m}$ & wo:mp $\theta$ & wo:m?p $\theta$ & warmth \\
\hline
\end{tabular}

(4) Rule A-B as a feeding relation:

a. Fortis Stop Insertion

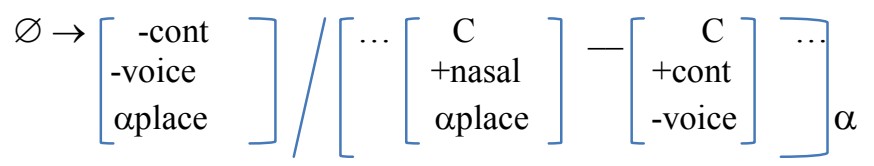

b. Preglottalization

$$
\left[\begin{array}{l}
\text {-cont } \\
\text {-voice }
\end{array}\right] \rightarrow[+ \text { constr }] /[+ \text { voice }] \text { _ }{ }^{(\# \#) ~ C} \quad \text { (Gussenhoven \& Jacobs, 2011, p.115) }
$$

Table 1 and the phonological rule in (4) illustrate phonological processes applying to the UR during their derivation as the SFs. It is shown that Fortis Stop Insertion rule feeds Pregottalization due to the phonological feature content that the affected environments have. These types of formalism will be employed for the rules affecting the derivation of Mengrelian verbs below. Where exactly at PF all types of phonological rules (cyclic vs. post-cyclic, morpheme-specific or other types) is not of concern here. These issues will further be addressed in future research with more empirical data at hand than the one presented in this paper.

\subsection{Megrelian preverbs}

There are thirteen preverbal markers in Mengrelian consisting of one syllable and often referred to as simple preverbs in traditional linguistics literature (Kajaia, 2001; Danelia, 1991). They can be added at the beginning of the multimorphemic template of verbs to mark directionality (if verbal roots indicate motion), perfective aspect or simply change the meaning of the Root into a completely new meaning. Here is the list of preverbal markers:

(5) a-, ga-, ge-, go-, da-, do-, e-, me- (//mi-), o-, she-, c'a-, c'o-

These markers may recombine with other 30 preverbal morphemes approximately reaching 150 combinations that are referred to as complex preverbs. The combinatorial possibilities of simple preverbs with other morphemes of their kin are much higher in Mengrelian than in Georgian, a sister language of South Caucasian family to which Mengrelian belongs. In Georgian, only two simple preverbs can combine at most (cf. Aronson, 1990). Here is the list of another 30 Mengrelian preverbal markers whose nucleus may vary from dialect one dialect to another. (note 2) Here we only reference the most typical phonological shapes of 30 preverbs:

(6) ato-, ako-, ala-, amo-, ano-, asha-, ac'o-, gato- gako-, gala-, gama-, gamo-, gano-, gasha-, gac'o-, dato-, dako-, dala-, dano-, gasha-, k'ala-, k'ano-, mada-, mato-, mak'o-, mala- mano-, masha-, mac'o-, c'amo-;

Note that these complex markers can incorporate into or recombine with other simple preverbs along with the assertive and negative markers $k^{\prime} o$ - and $v a$-. Sometimes the negative preverbal marker can combine with the assertive article $k^{\prime} o$ creating the following string at the beginning of the template:

(7)a. va-k'o-dziruo

$$
\begin{aligned}
& \text { Neg-Assert-see } \\
& \text { 'he did not even see.' } \\
& \text { b. va-k'o-mortuo } \\
& \text { Neg-Assert-come } \\
& \text { 'he did not even come.' }
\end{aligned}
$$$$
\text { (Kajaia, 2001, p.59) }
$$

It is also of interest that the vocabulary Item $-k^{\prime} o$ - may be inserted not only as an assertive marker but along with other five simple preverbal markers (-to-, $-l a-,-n o-,-s h a-$ and $\left.-c^{\prime} o-\right)$, it may recombine with other directionality markers to 
denote more precise localization in space (Kajaia, p.60). The preverb $-k$ ' $o$ - in such combinations means 'to be close to something, such as in mi-k'o-xuna 'to sit close to the wall, in corner', etc. (note 3).

It is also notable that some complex strings of preverbal markers may grow up to three and four morphemes that are referred to as super-complex preverbs in the literature (Kajaia, 2001; Xubua, 1942).

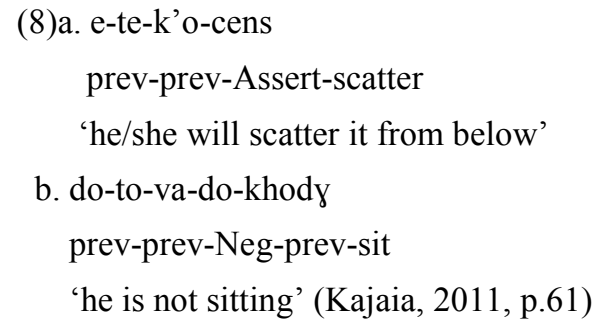

This information naturally leads to the question about the morpheme order in Mengrelian verbs which may be proposed based on Kajaia (2001) and Xubua (1942) as the following:

(9) Prev (2-3)-Neg-Assert- Subject/object clitic-voice/version-Root-Thematic marker...

The post-base component of this template is irrelevant here as the focus of this paper is the phonological processes applying to preverbal markers linearized to the left of Roots. The preverbal aspectual markers are at the beginning of the template followed by assertive/negative markers if present and subject/object agreement markers that resemble person clitics in Halle \& Marantz's (1993) sense occur only after the preverbal component. The version/voice markers may also be present when the structure includes the applicative $\mathrm{v}^{0}$ head that licenses the goal or destination argument in the clause. These morphological slots may add up before the Root, which in this account figures as one of the triggers of phonological rules along with other preverbal markers.

The rest of paper is organized as follows: Section 2.1 discusses morpheme-specific phonological processes such as dissimilatory deletion of certain vowels and voiced obstruents along with the metathesis rule affecting adjacent segments; Section 2.2. analyzes the rule triggered by the syllable structure constraints and section 2.3 discusses the series of opaque interactions applying in a block of at least three rules or more; Section 3.0 draws the conclusions and suggests future directions of research on morphophonolgical rules and their impact on the final form of complex expressions.

\section{Phonological rules as a source of allomorphic variety}

\subsection{Morpheme-specific phonological rules}

This section analyzes the phonological rules that are triggered by the addition of certain morphemes to the pre-base positions of the Mengrelian verb. As noted in section 1.2, the triggering morphemes are preverbal directionality markers that may be used to express the completed action denoted by the Root. Some of these preverbal elements can also be the assertive or negation markers. One general phonological process that affects the allomorphy of preverbal aspectual markers is Dissimilatory Deletion of vowels in the environment of Root as formulated in the following:

(10) Pre-Base Dissimilatory Deletion (PBDD)

Delete any vowel in preverbal markers which is identical with either the Root-initial or another preverbal vowel on its right.

The following example shows that the PBDD is active and affects the preverbal /o/ since the identical vowel shows up in the Root to the right of the preverbal marker. Crucially, the rule does not affect the Root vowel itself:

(11) Non-Front Vowel Dissimilatory Deletion (NFV) applies to string adjacent elements:

$$
\begin{aligned}
& \text { a. / o / } \left.\rightarrow \varnothing / \_\sqrt{ } \text { Root[o- }\right] \\
& \text { b. mi-k'o-v-odzni } \rightarrow \text { mik'-v-odzni } \\
& \text { prev-prev-1S-look } \\
& \text { 'I looked at' (Kajaia, 2001, p.60) }
\end{aligned}
$$

There is one interfering morpheme between the syllables with the identical nuclei in this word, and that is the subject clitic $v$ - that has no effect on the neighboring vowels. Note that in the third person where the subject clitic is zero, the two vowels create hiatus and this hiatus is removed by the deletion of the vowel in the preverbal marker. It can be concluded that the phonological rule in (11) targets not morpho-syntactic constituency but identical phonological segments of the terminal nodes that merge higher in the tree than the Root itself. (Note 3)

The similar Dissimilatory Deletion rule may target the high front vowel $i$ - in preverbs in the environment of the identical vowel in the Root. It is of importance to observe that these identical vowels are not string adjacent (SA) but are separated by another preverbal marker c'o-:

(12) High Vowel Deletion (HVD)

$$
\begin{aligned}
& \text { a. } \mathrm{i} \rightarrow \varnothing / \ldots \text {. }{ }^{\sqrt{i}} \longrightarrow \\
& \text { b. ge-gi-c'o-iyans } \rightarrow \text { ge-g-c'o-iyans }
\end{aligned}
$$


prev-prev-prev-take

'he/she will take it from you' (Kajaia, 2001, p.60)

Note that the UR of this word should contain the preverbal gi- element as a part of the verbal meaning and the supersized preverbal marker but after gi- is added to the pre-base component the rule in (10) is activated and the deletion of the identical vowel in the preverb follows. The feature content of the vowel is irrelevant here whether it is high front or mid back vowel: any vowel can undergo deletion due to the rule in (10). The fact that the deletion happens not in the Root but outside of it indicates that the phonological processes apply cyclically and the Roots are realized in a different cycle than preverbal markers that are added in the derivation later.

Another Dissimilatory process that targets not only identical vowels but also consonants in the preverbal markers is the one that deletes the velar stop /g/ from preverbal markers in (12). However, when the velar stop is targeted for deletion, the identical vowels are kept until the further change affects them. In the following, the series of phonological rules affecting the UR is shown:

(13) Velar Stop Deletion (VSD):

$$
\begin{aligned}
& \text { a. } g \rightarrow \varnothing / C_{[\mathrm{G}]} \mathrm{V} \text {. } \\
& \text { b. ge-gi-c'o-iyans } \rightarrow \text { ge-i-c'o-iyans }
\end{aligned}
$$

This change is not attested in the same dialect of Mengrelian as (12) and the hiatus formed as a result of its application is removed by the Glide Formation rule that will be discussed in the next section where other rules motivated by the syllable structure constraints are also discussed:

(14) Glide Formation Rule (GF):

$\mathrm{ei} \rightarrow \mathrm{ej} / \mathrm{CV}^{\prime}$.

The reduction of the second vowel to the glide happens due to the stressless syllable in which $-i$ occurs. The GF does not apply in the same word where the Root-initial and the preverbal vowel $o$-create hiatus and this happens because the Root vowel receives secondary stress and it is not reduced to the glide in that environment.

The same series of phonological changes is illustrated in another form as well:

(15) VSD rule applies:

$$
\begin{aligned}
& \text { a. } \mathrm{g} \rightarrow \varnothing / \mathrm{C}_{[\mathrm{G}]} \mathrm{V} . \\
& \text { b. ge-gi-to-uxotans } \rightarrow \text { ge-i-to-uxotans } \\
& \text { prev-prev-prev-throws }
\end{aligned}
$$$$
\text { 'he/she throws it up from below' (Kajaia, 2001, p.61) }
$$

(16) GF rule applies afterwards:

ge-i-to-uxotans $\rightarrow$ gej-to-uxotans

(17) NFV Deletion applies:

$$
\text { gej-to-uxotans } \rightarrow \text { gej-t-uxotans }
$$

It can be concluded that VSD and GF rules apply cyclically and the VSD rule in (15) is in feeding relation with the GF rule. The resulting word may not keep the initial meaning of the unreduced preverbal markers which indicates that the phonological changes induce the rules in the meaning of nested constituents computed in syntax. For example, in (15) the meanings of individual preverbal markers like ge-, gi-, and to- are not all traceable in the SF. 'To-' means 'located in remote deep place' and it is not evident in the SF as well as that of ' $g i-$ ' which is reduced to ' $g$-'. This evidence poses some questions for the derivational theory like DM which proposes that the sensory-motor and the conceptual interfaces of language are independent of each other as illustrated in the figure1. This view should be modified if the data presented here will be supported with more empirical evidence from other languages.

All in all, the rules discussed in this section are morpheme-specific as they apply only to preverbal markers in the environment of Roots, and they are cyclic applying to nested constituents.

\subsection{Syllable-specific phonological rules}

The interaction of cyclic and post-cyclic phonological rules can sometimes obscure the SF of the derived words especially if several cyclic rules apply initially, and then they are followed by the post-cyclic one rule applying to the entire word. The following illustrates the application of several deletion rules followed by the post-cyclic Metathesis, which is also motivated by the syllable structure constraints:

(18) do-mi-sha-no-yalue(n) $\rightarrow$ dimsh-no-yalue(n) (Surface Form) (Note 4)

prev-prev-prev-prev-carry

'He used to carry it inside'

It can be argued that the rule of Non-High Vowel (NHV) Deletion applies to non-SA elements first and then it is followed by another Dissimilatory Deletion rule affecting the non-front identical vowel in the preverbal marker at the beginning of the word: 
(19) NHV Deletion:

$\mathrm{a} \rightarrow \varnothing / \ldots$ CV.C[a]

do-mi-sha-no-yalue(n) $\rightarrow$ do-mi-sh-no-yalue(n)

After (21), NFV Deletion rule applies skipping one syllable:

(20) o $\rightarrow \varnothing / \_$CV. C[o]

do-mi-sh-noyalue $(\mathrm{n}) \rightarrow$ d-mish-noyalue $(\mathrm{n})$

The post-cyclic Metathesis rule applies to the output of Deletion rules, and it is presumably motivated by the syllable structure constraints banning the consonant cluster like $d m$ at the beginning of the Word:

(21) Metathesis rule:

d-mi-sh-noyalue(n) $\rightarrow$ dim-sh-noyalue(n)

Note that the metathesis rule does not affect the meaning of the preverbal markers and the idea here is that the remnant from the preverbal marker sha-/she- is reanalyzed as an initial segment of the next syllable resulting in the consonant cluster at the beginning of the second syllable. The meaning of 'sha-' between, inside is kept in the surface form but that of ' $d o$ '- and ' $m i$ ' - is not. The Metathesis rule applying post-cyclically does not change meaning. It can be concluded that the phonological changes at PF affect the meaning of the derived words and this type of interaction between meaning and phonological processes is not possible in the architecture of grammar given in (1).

Another similar example of the series of rules involve the assertive marker ' $k o$ '- that can be added either at the beginning of the word or even after the aspectual markers as mentioned in Section 2.1. The SF is still recognizable from its UR that is the interaction of the phonological rules is transparent:

(22) k’o-e-te-mo-cens $\rightarrow$ k'et-mo-cens

Assert-prev-prev-prev-scatter

'It is even scattered from below upwards' (Kajaia, 2001, p.61)

Like in (18), the derivation of the SF involves Dissimilatory Deletion rules in (19) and (20) that apply to the string adjacent and non-tring-adjacent phonological exponents resulting in the form ktemocens that is subsequently affected by the Metathesis rule as in (21). Here are these changes that are in feeding relation from (23) to (25):

(23) Non-High Vowel Deletion (NHV Deletion):

a. $\mathrm{e} \rightarrow \varnothing$

b. k'o-e-te-mo-cens 'throw up from below' $\rightarrow$ k'o-te-mocens

(24) Dissimilatory NFV Deletion (as in (5)) skipping a morpheme:

a. $\mathrm{o} \rightarrow \varnothing$

b. k'o-te-mo-cens $\rightarrow$ k'te-mo-cens

(25) Metathesis applying to string adjacent elements:

k'te-mo-cens $\rightarrow$ k'et-mo-cens

It should be noted though that the phonological processes described above do not involve any morpho-syntactic features of the nodes that are realized during the VI with the phonological material. The phonological rules affecting such features do exist in Mengrelian and they primarily affect the subject and object clitics in certain phonological environments but their exact nature is a topic of another paper and will not be discussed here. As shown in (9), the subject/object clitics which have only one slot for realization in the pre-base component of the template follow preverbal aspectual markers and precede voice/version morphemes which are mainly vowels. Like aspectual/directionality markers, the clitics may also be deleted resulting in the zero marking of certain arguments in the clause. The main conclusion drawn from this section is that when multiple preverbal markers accumulate at the beginning of the verb, they are invariably affected by the morpheme-specific Deletion rules like in (10) followed by post-cyclic rule that applies just once without any impact on the meaning of the resulting word. This empirical observation lends supports to the derivational view of morphology and phonology as well but the architecture of grammar according to DM should be modified to support these facts.

\subsection{Assertive and progressive markers and opaque interactions between rules}

This section shows the derivation of the surface form with the changes that go beyond the more systematic Dissimilatory Deletion rule like the one in (10). Some rules here are slightly different than those shown in the preceding sections although they interact with the rule in (10). The cases covered in this section create two different outcomes in terms of the opacity of the SFs, the first outcome being opaque forms derived through self-destructive feeding relationship while the second, transparent forms. Here is the illustration of opaque forms with their UR and SF of the derived word:

(26) k'o-mik'o-ipkuni $\rightarrow$ k'i-m-k'-ipkuni (Surface Form)

Assert-prev-put

'I even put it on' (Kajaia, 2001, p.62) 
It can be hypothetically proposed that the SF in (26) is derived through the application of a number of phonological rules affecting the UR, and the first rule is Non-Front Vowel Deletion triggered by the identical vowel on the right:

(27) NFV Dissimilatory Deletion applies to the left:
a. $\mathrm{o} \rightarrow \varnothing /$ CV.CV[o]
b. k'o-mik'o-ipkuni $\rightarrow$ k'-mik'o-ipkuni

This dissimilatory deletion may reapply due to the hiatus created by the preverbal marker mik'o- and the Root-initial vowel. The result is a complete removal of the NFV environment from the word:

(28) Another NFV Deletion:

a. o $\rightarrow \varnothing / \_$V[i]

k'-mik'o-ipkuni $\rightarrow$ k'-mik'-ipkuni

The final rule will apply post-cyclically and it will only affect the phonological form of the entire word:

(29) Post-cyclic Metathesis:

k'-mik'-ipkuni $\rightarrow$ k'im-k'-ipkuni (SF)

Note that the final form is deeply obscured with respect to the UR shown in (26) and it is clear that the SF is derived through the changes that involve the self-destructive feeding relation between the rules like (27) and (28). The formal description of this rule as explained in Bakovič (2007) and Arregi \& Nevins (2012) is the following:

(30) Self-Destructive Feeding

The two rules are in self-desructive feeding relation when the rule B applies at the "expense" of another rule A, i.e. the application of the rule B after the rule A causes the destruction of the environment in which A applied.

At the same time, the environment in which (27) applies is destroyed when (28)-(29) apply to the intermediate representations. The SF is substantially obscured with respect to UR.

In other cases, the result may not be opaque as in (31). For example, the preverbal markers may be expanded by the progressive markers like tima-, or ti- and $m a$ - that can be carried over throughout the Present series screeves of Mengrelian. (Note 5) By adding these progressive markers, the series of Dissimilatory Deletion rules may apply along with the simple deletion rules like in the following forms:

(31) a. e-sha-ma-dinu(n) $\rightarrow$ eshmadinu (SF)

prev-prev-prev-loose

'it is being lost' (Kajaia, 2001, p.60)

b. ge-tima-igonens $\rightarrow$ getmigonens (SF)

prev-Progr-hears

'he even hears it' (Kajaia, 2001, p.60)

The addition of the progresive marker $m a$ - in (31b) triggers the general Dissimilatory Deletion rule like in (10) to apply resulting in the shorter Surface Form that is lacking the identical nuclei in the two syllables. Observe that the nucleus of the syllable on the left of the triggering enviroment is deleted rather than in the preverb, which comes to the right of the progressive marker $m a$-. However, the addition of the progressive prefix tima- triggeres several rules to apply:

(32) PBDD applies to the UR:

ge-tima-igonens $\rightarrow$ ge-tma-igonens

As the result of this rule, the hypermetaphony raises /a/ to /i/ after the application of the rule in (32). This is an assimilation of the low vowel to a high one $/ \mathrm{i} /$ :

(33) Hypermetaphony:

a. $a \rightarrow \mathrm{i} / \ldots$. V $[\mathrm{i}]$.

b. ge-tma-igonens $\rightarrow$ ge-tmi-igonens

It is evident that the two identical vowels in (33) is not tolerated due to (10) and the preverbal vowel is deleted resulting in the surface form:

(34) HVD applies repeatedly:

ge-tmi-igonens $\rightarrow$ ge-tm-igonens

It can also be argued that the same environmental triggers cause two different rules such as PBDD and Hypermetaphony in (31b), and (32) and (33) are in feeding relationship with each other as well as (33) and (34). However, these rules do not result in an opaque form like the changes in (29) and the SF can be predicted from UR. Thus, the series of phonological rules create optimal phonological strings in the resulting forms while the meanings of individual morphemes are affected with the series of cyclic rules, which are morpheme-specific. 


\section{Conclusions}

The paper showed the phonological processes affecting the derivation of verbal expressions in Mengrelian may not only obscure the surface forms from underlying representations but more importantly the meanings of some of the preverbal morphemes undergoing the phonological changes can also be modified. The evidence shown in this paper supports the view that cyclic phonological processes affect the meaning of the derived words but post-cyclic processes which apply to an entire word do not. It is concluded that the current view of DM accommodating completely independent LF and PF components should be modified by bringing more evidence to the existing data provided in this paper.

\section{References}

Aronson, H. (1990). Georgian: A reader's grammar. Columbus, OH: Slavica Publishers.

Arregi, C. \& Nevins, A. (2012). Structure of Spellout. Morphotactics of Basque Auxiliaries. Dordrecht: Springer.

Bacovič, E. (2007). A revised typology of opaque generalizations. Phonology, 24, 214-259.

Danelia, K. (1991). The issues in the lexicology of the Mengrelian-Chan language. Georgian Folklore, Mengrelian texts. Tbilisi: Tbilisi State University Press.

Chomsky, N. \& Halle, M. (1968). Sound pattern of English. New York: Harper \& Row.

Gussenhoven, C. \& Jacobs, H. (2011). Understanding phonology. Third edition. London, UK: Hodder Education.

Halle, M. \& Marantz, A. (1993). Distributed Morphology and pieces of inflection. In View from building, 20, 111-176. Cambridge, MA: MIT Press.

Halle, M. \& Mohanan, K.P. (1985). Segmental phonology of Modern English. Linguistic Inquiry, 16, 57-116.

Halle, M. \& Matushansky, O. (2006). The morpho-phonology of Russian adjectival inflection. Linguistic Inquiry, 37, 351-404.

Halle, M. \& Nevins, A. (2009). Rule application in phonology. In E. Raimy \& Ch. Cairns (eds.), Contemporary Views on Architecture and Representations in Phonology, 355-383. Cambridge, MA: MIT Press \& London, UK: Bradford Books.

Kajaia, O. (2001). Megrul-Kartuli leksikoni. [Mengrelian-Georgian dictionary]. V.1, Tbilisi, Georgia: Nekeri.

Kenstowicz, M., and Kisseberth, Ch. (1979).Generative phonology. San Diego: Academic Press.

Kenstovicz, M. (1994a). Phonology in Generative Grammar. Oxford: Blackwell.

Kiparsky, P. (1982). Word formation and the Lexicon. In F. Igemann, (ed.), Mid-American Linguistics conference papers, 3-29. Department of Linguistics, University of Kansas, Lawrence.

Verkuyl, H. (1989). Aspectual classes and aspectual composition. Linguistics and Philosophy, 12, 39-94.

Xubua, M. (1942). The assertive particle k'o- in Mengrelian, The Bulletin of the Academy of Sciences of Georgia, Tbilisi, Georgia: Metsniereba [Academic Press].

\section{Notes}

Note 1. This hypothesis is based the more recent research on PF processes (Harris and Halle 2005, Arregi \& Nevins, 2012 among others) that argues for the separation of Linearization from VI since the processes affecting the morpheme order such as reduplication and metathesis happen after Linearization before VI takes place. This paper therefore argues that the possible location of the phonological rules affecting various morphological domains like nested constituents and entire words should be following VI.

Note 2. In this paper, all data comes from Zugdidi and Suxumi dialects of Mengrelian, the former primarily illustrated by Kajaia's grammar sketch at the beginning of the Megrelian-Georgian Dictionary, and the latter by the native speaker consultant whose judgments are scattered throughout the paper.

Note 3. This type of flexibility of the Vocabulary Item like that of $-k$ ' $o$ - can be explained with the underspecification of these items with respect to the nodes in which they are inserted. Note that the notion of underspecification is one of the most important ones in DM.

Note 4. It is not evident from the existing data that there is a language-specific syllable structure constraint banning any hiatus and repairing such vowel strings with some phonological rule. However, (12) is clearly illustrated in the data at hand.

Note 5. Native speakers of the Suxumi dialect of Mengrelian prefer the form without the initial /d/ as in the following: mishnoyalue(n) as opposed to the speakers of Zugdidi dialect illustrated by the example from Kajaia (2001).

Note 6. Series represent the conjugation paradigms of verbs with distinct Tense-Mood-Aspect (TMA) features like in the sister language Georgian and in Present series, the series and screeve markers may show up in the pre-base sector asopposed to Georgian where TAM markers show up at the end of the verbal template.

Note 7. It would be tempting to posit the Deletion rule first for the nucleus of $m a$ - but there is no motivation for such deletion while for the preverbal $i$ - in tima- there is clearly such motivation because of the identical Root-initial vowel. 